\title{
Temporary Internal Iliac Artery Blockage versus Uterine Artery Embolization in Patients After Laparoscopic Pregnancy Tissue Removal Due to Cesarean Scar Pregnancy
}

\author{
Qian Zhao* \\ Xue-Yu Sun* \\ Shu-Qi Ma \\ Meng-Wei Miao \\ Gai-Ling Li \\ Jing-Lu Wang \\ Rui-Xia Guo \\ Liu-Xia Li
}

Department of Gynecology, The First Affiliated Hospital of Zhengzhou University, Zhengzhou, 450000, People's Republic of China

*These authors contributed equally to this work
Correspondence: Rui-Xia Guo Department of Gynecology, The First Affiliated Hospital of Zhengzhou University, No. I of Jianshe Dong Street, Erqi District, Zhengzhou, 450000,

People's Republic of China

Tel +86 I3525569376

Email guoruixia70@163.com
Objective: This study aims to evaluate the clinical value of laparoscopic temporary internal iliac artery blockage (TIIAB) compared with uterine artery embolization (UAE) in type III cesarean scar pregnancy (CSP).

Methods: A total of 76 patients with type III CSP admitted to the Department of Gynecology the First Affiliated Hospital of Zhengzhou University between September 2017 and June 2019 were selected for this retrospective study. Thirty-six of them in the study group received TIIAB, and the rest in control group received UAE. Laparoscopic pregnancy tissue was removed from all patients, and the uterine defects were repaired. The absence of remnants was then confirmed using ultrasonography. Follow-ups were performed in the two groups for six months, and the factors of intraoperative blood loss, operation and menelipsis time, 24-h human chorionic gonadotropin decline rate, postoperative complications, hospitalization days, hospitalization costs, peri-operative hormone levels, and ovarian function indicators were compared between the two groups and within each group.

Results: There were statistically significant differences in the hospitalization cost, menelipsis time, and postoperative complication incidence between the two groups $(\mathrm{p}<0.05)$. There were statistically significant differences between ovarian function at one month and three months after surgery $(\mathrm{p}<0.05)$ as well as among the follicle-stimulating hormone, luteinizing hormone, and estradiol levels at one, three, and six months after surgery in the control group $(\mathrm{p}<0.05)$.

Conclusion: Compared with uterine artery embolization, laparoscopic TIIAB has the advantages of a low hospitalization cost, lower postoperative complication rate, and shorter menelipsis time. Moreover, it avoids ovarian function damage. It is a safe method worthy of clinical popularization.

Keywords: type III cesarean scar pregnancy, laparoscopic internal iliac artery, occlusion, uterine artery embolization, ovarian function

\section{Introduction}

Cesarean scar pregnancy (CSP) refers to a special type of ectopic pregnancy featuring gestational sacs, fertilized eggs, or embryos implanted on the lower uterine segment cesarean incision scar in a pregnant woman with a history of cesarean section. It is a long-term cesarean section complication. ${ }^{1}$ CSP has attracted great attention in clinical practice due to its severe complications, such as massive bleeding, uterine rupture, peripheral organ damage, and even hysterectomy, which 
is difficult to control and is a serious threat to women's reproductive health and even their lives. ${ }^{2}$ The CSP concept was first reported by Larsen et $\mathrm{al}^{3}$ in 1978; however, only 19 additional cases were reported between 1978 and 2001. Nevertheless, a large number of case reports and metaanalyses have been conducted on CSP treatment and management practices since $2001 .{ }^{4}$ CSP incidence is low in China, with approximately 1 per 1800-2216 pregnant women, which accounts for $0.15 \%$ of all pregnancies. ${ }^{5}$ Although an increased recurrent CSP morbidity can be expected to increase after the first cesarean section occurrence, greater awareness and enhancement of CSP clinical diagnostic techniques among obstetricians and gynecologists can also contribute to the rate increase. ${ }^{4-8}$ Although the CSP pathogenesis is presently unclear, the most prominent theory on the matter is that the pathogenesis is closely related to a muscular layer defect caused by posttraumatic wound healing damage leading to localized depression. ${ }^{9}$ There is still a lack of unified CSP treatment guidelines. Individual treatment plans should be made based on the comprehensive consideration of the patient's blood $\beta$-HCG level, scar site muscular thickness, mass size, blood flow, clinical symptoms, and own wishes. Currently, commonly used treatment methods include drug therapy, surgical treatment, or a combination of the two. Fylstra ${ }^{10}$ reports that CSP resection + uterine repair may be the best CSP treatment method. The Society for Maternal-Fetal Medicine also suggests that surgical resection (transvaginal or laparoscopic surgery if possible) should be considered for patients with CSP. ${ }^{11}$ Not only can this method terminate pregnancy effectively, but it can also repair scar defects as effectively as possible, which is conducive to uterine physiological function recovery. However, one-time treatment may not achieve the ideal therapeutic effect in patients with a high risk of bleeding. For this reason, effective pretreatment before the laparoscopic removal of pregnancy lesions should be conducted in order to reduce the risks of intraoperative and postoperative massive bleeding. One of the classic CSP pretreatment methods is uterine artery embolization (UAE). It is widely used as a minimally invasive method in clinical practice. However, the reported adverse reaction incidence is $8.0-51.7 \%{ }^{12}$ and treatment-related complications include large-area uterine necrosis, postoperative pain, pelvic infection, local bladder or rectum necrosis, premature ovarian failure, and amenorrhea. ${ }^{13}$ Simultaneously, the high cost of surgery and interventional instruments, radiation exposure of both the patients and medical personnel long after the treatment, and the time lag between embolization and follow-up surgery make UAE a non-optimal choice in non-emergency cases.

Based on clinical experience, temporary internal iliac artery blockage (TIIAB) has become one of the pretreatment methods. It is conducted in accordance with the specific conditions of patients with type III CSP. If local lesion resection is followed by bilateral internal iliac artery ligation, the risks of intraoperative and postoperative bleeding can be significantly reduced. ${ }^{14}$ Lots of researches have been reported since Dubois first reported a case series about invasive placentation in which balloon occlusion and embolization of the internal iliac arteries (IIA) were used to prevent postpartum hemorrhage. ${ }^{15}$ Due to the high risk of bleeding in III CSP, we definitely should consider the bleeding risk. Thinking of the lasting effect from the surgery people with III CSP have to suffer, the volatility of ovarian function after the surgery has attracted more and more attention. Due to all the mentioned above, the aims of this study are:

To evaluate the clinical value of laparoscopic TIIAB compared with UAE in type III CSP, and to further explore the safety and clinical promotion prospects of TIIAB.

\section{Materials and Methods \\ Subjects}

We have researched all CSP patients through the medical record system and screened them by inclusion criteria, a total of 76 patients with type III CSP admitted to the Department of Gynecology the First Affiliated Hospital of Zhengzhou University between September 2017 and June 2019 were selected for this retrospective study. patients with type III uterine scar pregnancy correspond with the diagnosis standards of China's Expert Consensus on Diagnosis and Treatment of Uterine Scar Pregnancy after Cesarean Section (2016). ${ }^{2}$

\section{Inclusion Criteria}

Inclusion criteria: (1) patients with consent in reproductive age (2) patients with normal ovarian function (Hormone levels during menstruation) and no family history of premature ovarian failure; (3) patients with good compliance who regularly attended re-examination; (4) TypeIII (the gestational sac is completely implanted in the muscularis at the scar of the uterus and convex to the bladder direction; uterine cavity and cervical canal empty; the myometrium between the gestation sac and the bladder was thinned or even missing loss, thickness $\leq 3 \mathrm{~mm}$;) according 
to 2016 Chinese Medical Association classification needed surgery. (5) The state of an illness is stable and patients can wait for the scheduled surgery. (6) The patient has been fully informed of the alternatives and risks associated with the operation and signed the informed consent for the operation.

\section{Exclusion Criteria}

(1) Patients with severe organ dysfunction; (2) patients with severe coagulation function disorders; (3) patients with surgical contraindications; (4) patients with uterine malignancy or other diseases requiring an extended surgical scope; (5) patients with other systemic malignancies or severe infections who cannot tolerate laparoscopic surgery; and (6) patients with an open abdominal cavity transfer during surgery.

\section{Grouping}

The patients who met the above-listed criteria were divided into two groups according to the different intervention: the study group $(\mathrm{n}=36)$ and the control group ( $\mathrm{n}$ $=40$ ). Patients who received TIIAB and underwent laparoscopic pregnancy tissue removal were assigned to the study group, while patients who received UAE and underwent laparoscopic pregnancy tissue removal were assigned to the control group.

\section{Test Methods}

The fixed interventional therapy team in our hospital who performed the UAE were all experienced and had obtained professional certificates of interventional physicians and senior professional titles, mastering pelvic vascular anatomy. The invasive radiology team are included in the group for the measurement or UAE.

\section{Study Group Surgical Methods}

The specific surgical methods for laparoscopic TIIAB combined with pregnancy tissue removal were as follows. Under general anesthesia, the patient taking bladder cut stone was placed on the operating table The indwelling urinary catheter was regularly disinfected and draped, the Trocar was placed along the margin of the transverse incision $(1 \mathrm{~cm})$ above the umbilicus and used to fill the cavity with $\mathrm{CO} 2$. After the artificial pneumoperitoneum formation, a cavity mirror was put in place. It was used to monitor the procedure, and two incisions were made: (1) an incision with $1 \mathrm{~cm}$ inside the left anterior superior spine (c. a finger's width) and (2) an incision with $0.5 \mathrm{~cm}$ outside the middle point of the line between the former incision and umbilicus (c. $1 \mathrm{~cm}$ ). The respective Trocars were placed. On the right side of the McIntosh point, another $1.2-\mathrm{cm}$ incision was made, and the was Trocar placed. The intraoperative observations were as follows: the muscle layer under the anterior wall of the lower uterus segment was paper thin and obviously visible using the pregnancy convex. The muscle layer had a rich blood supply. The ureter was discerned, and the retroperitoneum was opened, freeing the initial iliac and uterine artery parts and temporarily blocking the middle of the bilateral iliac artery with ligated No. 7 silk slipknots. Next, the pregnancy tissue removal surgery and uterus repair were carried out. The bladder was opened via the peritoneal reflex and pushed down (c. $1.5 \mathrm{~cm})$. After cutting open the cesarean section scar through the full uterus thickness using a unipolar electric hook, a large amount of burgundy-colored pregnancy tissue and hemorrhage blocks could be seen. The tissue was taken out through the right lower quadrant abdominal incision. The cesarean section scar and the weak uterine wall were pruned until thicker, fresh uterine wall tissue could be seen. The uterus incision was then sutured continuously with an absorbable line, and the uterus morphology and normal tissue layers were restored. Next, the slipknots on both sides of the iliac artery were opened and observed for five minutes. If the patient had normal blood pressure and no active uterine bleeding, the operation was ended. The operation is shown in Figure 1.

\section{Control Group Surgical Methods}

The specific operation methods for laparoscopic UAE combined with pregnancy tissue removal were as follows. With the patient in the supine position on the operating table, bilateral inguinal areas were disinfected and draped routinely under local anesthesia. The right femoral artery was successfully punctured using the Seldinger method, and the vascular sheath was introduced. Next, five pigtail catheters were introduced at waist level 2 high to image using high pressure, and the bilateral uterine artery was obviously enlarged, with a rich blood supply observable inside the pelvic cavity. The uterus abnormal be stained obviously in substantial period, and the ovarian arterial could not be developed. The head ends of 0.035 -inch Cobra catheter hydrophilic membrane guide wires were inserted into the bilateral internal iliac arteries and uterine arteries for angiography, along with double uterine arteries on each side. The uterine arteries were super-selected and embolized with five bottles of 350-550-um gelatin sponge 


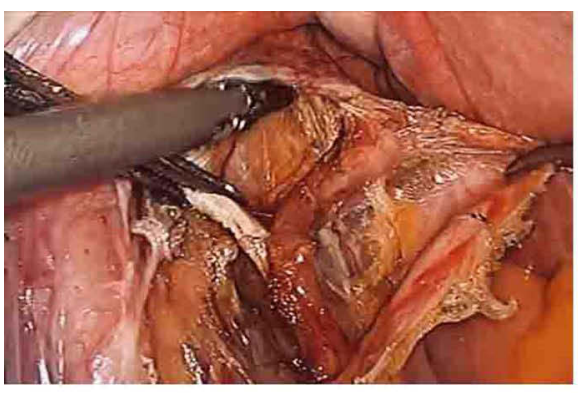

A

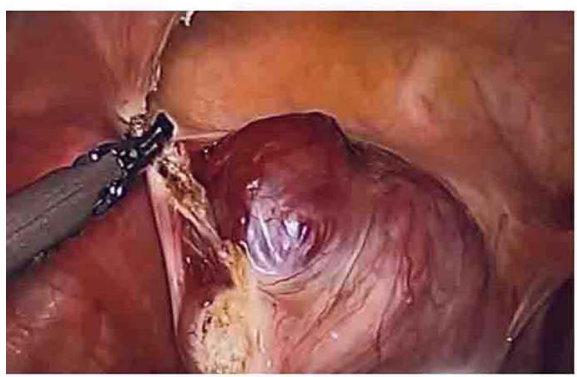

C

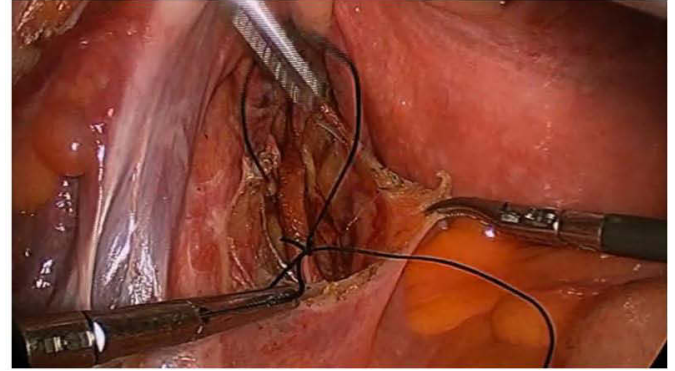

B

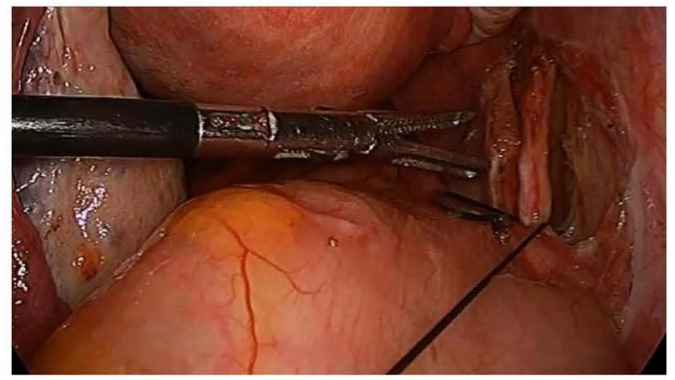

D

Figure I Laparoscopic TIIAB. (A) Left internal iliac artery anatomy. (B) Internal iliac artery slipknot (No. 7 silk) ligation. (C) CSP cite. (D) Slipknot unlocking after pregnancy tissue removal.

particles and gelatin sponge with hand clipping until the blood flow was essentially blocked. The catheter sheath was pulled out, local pressure was applied to stop the bleeding, and pressure bandaging was performed before the patient returned to the ward. If no obvious abnormity was found after a 24-h rest, the patient was sent to the operating room for laparoscopic hysterotomy and pregnancy tissue removal + uterine repair under general anesthesia. After the routine establishment of artificial pneumoperitoneum, the laparoscopic instruments were put in place, and the pelvic and abdominal conditions were explored. Then, the hysterotomy and the pregnancy tissue removal + uterine repair were performed; the steps were the same as written above.

\section{Discharge Criteria}

The same discharge criteria applied to both groups. Discharge criteria: (1) the blood $\beta$-human chorionic gonadotropin (HCG) level had decreased by $>50 \%$; (2) a normal diet was resumed and there was no need for intravenous infusion; (3) the wound had healed well and there were no signs of infection; (4) organ function was good; and (5) the patient could move freely and agreed to be discharged.

\section{Follow-Up Methods}

All patients attended follow-ups for six months after the procedure via outpatient reexamination and telephone contact, and inquiries were made regarding the patients' menstrual cycles and clinical symptoms. The follow-up period was until May 31, 2019.

\section{Observation Indicators}

\section{Intraoperative and Postoperative Conditions}

The operative time, intraoperative blood loss, serum $\beta$-HCG percentage decrease in $24 \mathrm{~h}$ after surgery, time before menstruation resumption, total length of postoperative hospital stay, hospitalization cost, and postoperative complication incidences (pelvic pain, fever, venous thrombosis, and intrauterine adhesion) were observed in the two groups.

\section{Ovarian Function Evaluation}

Preoperative blood was drawn for ovarian endocrine function examination. On the 2-5th day of the primary menstrual cycle, blood samples were collected to check the follicle-stimulating hormone (FSH), luteinizing hormone (LH), and estradiol (E2) levels in the nuclear medicine department of our hospital at one, three, and six months after surgery (the same hospital and nuclear 
Table I Comparison of Baseline Data Between the Two Groups $(\overline{\mathrm{x}} \pm \mathrm{s})$

\begin{tabular}{|c|c|c|c|c|}
\hline Indicators & The Study Group $(n=33 / 36)$ & The Control Group $(n=35 / 40)$ & $t$ & $\mathbf{P}$ \\
\hline Age (year) & $28.97 \pm 4.61$ & $29.03 \pm 4.19$ & -0.50 & 0.96 \\
\hline Menelipsis time (day) & $48.51 \pm 2.39$ & $48.91 \pm 2.20$ & -1.39 & 0.17 \\
\hline Blood $\beta$-hCG Before operation ( $\mathrm{mlU} / \mathrm{mL}$ ) & $|8,906.12 \pm| 1,296.74$ & $17,852.57 \pm 10,585.35$ & 0.42 & 0.68 \\
\hline Times of cesarean section & $1.56 \pm 0.65$ & $1.65 \pm 0.70$ & -0.61 & 0.55 \\
\hline Operation time $(\mathrm{min})$ & $|26.47 \pm| 3.2 \mid$ & $125.58 \pm 22.96$ & 0.21 & 0.84 \\
\hline Intraoperative blood loss (mL) & I I $2.44 \pm 22.65$ & $102.22 \pm 22.91$ & 1.94 & 0.06 \\
\hline Menstruation length(d) & $4 \pm 0.23$ & $4 \pm 0.49$ & -0.19 & 0.05 \\
\hline Menstruation regularity(d) & $28 \pm 1.11$ & $26 \pm 1.69$ & 3.67 & 0.33 \\
\hline PCOS rate(\%) & $0.08 \pm 0.20$ & $0.06 \pm 0.30$ & 3.31 & 0.72 \\
\hline Gravidity & $2.72 \pm 0.31$ & $2.06 \pm 1.86$ & -0.93 & 0.64 \\
\hline Parity & $1.75 \pm 0.52$ & $1.82 \pm 0.63$ & 2.68 & 0.07 \\
\hline Abortion times & $1.02 \pm 0.83$ & $1.35 \pm 0.53$ & 2.83 & 0.05 \\
\hline
\end{tabular}

Abbreviation: PCOS, polycystic ovary syndrome.

medicine laboratory). Furthermore, an ultrasonography was performed to monitor the sinus ovarian follicle number at the corresponding period.

\section{Statistical Methods}

The Statistical Package for Social Sciences 22.0 was used for processing data, with the normality of continuous variables was tested by $\mathrm{K}-\mathrm{S}$ test. Normally distributed measurement data were expressed as mean \pm standard deviation (SD), while non-normally distributed measurement data were expressed as median (P25, P75), and the comparisons were examined by Student- $t$ test and MannWhitney test (non-parametric distribution). The $\chi^{2}$ test was used for rate comparisons. The test level was 0.05 , and a $\mathrm{p}$ value of $<0.05$ was considered statistically significant.

\section{Results}

During the follow-up period, 2 patients in the study group forgot the bad sex hormones 6 months after menstruation, and 1 patient was lost to follow-up. In the observation group, 2 patients were not tested for sex hormones 3 months after surgery, and 3 patients were not tested for sex hormones 6 months after surgery.

\section{Baseline Data Comparison Between the Two Groups}

The age, amennorhea day number, serum $\beta$-HCG value at admission, and cesarean section times were respectively compared between the two groups; the differences were not statistically significant $(\mathrm{P}>0.05$, Table 1$)$.

\section{Relevant Perioperative Follow-Up Indicator Comparison Between the Two Groups}

The operation time was calculated from the beginning of the laparoscopic surgery (excluding the interventional surgery time). The respective operation time and intraoperative blood loss comparisons were made between the two groups; the differences were not statistically significant ( $\mathrm{P}$ $>0.05$ ). However, the amount of intraoperative blood loss was slightly lower in the control group than in the study group $(\mathrm{P}=0.06)$ (Table 1).

\section{Postoperative Rehabilitation Indicator Comparison Between the Two Groups}

The respective 24-h postsurgical serum $\beta$-HCG decrease rate and length of hospital stay comparisons were made between the two groups; there were no statistically significant differences $(\mathrm{P}>0.05)$. The respective hospitalization cost $(\mathrm{P}<$ $0.001)$, menelipsis time $(P=0.001)$, and postoperative complication incidence comparisons $(\mathrm{P}=0.036)$ were made between the two groups; the differences were all statistically significant $(\mathrm{P}<0.05)$. In the study group, only 2 patients $(6 \%)$ had postoperative fever, while in the control group, 3 patients (7.5\%) had postoperative fever, 5 (12.5\%) experienced pelvic pain, and $1(2.5 \%)$ had venous thrombosis in the lower right extremity veins. The total postoperative complication rate was $22.5 \%$. Low molecular heparin calcium (specification: $4 \mathrm{~mL}$ : 4100AXaIU/piece, injection, national medicine approved H20063910, Hebei Changshan Biochemical Pharmaceutical Co., Ltd., China) was administered in the two groups for antithrombotic treatment after surgery (Table 2). 
Table 2 Comparison of Postoperative Rehabilitation Indicators Between the Two Groups

\begin{tabular}{|c|c|c|c|c|}
\hline Indicators & The Study Group ( $n=33 / 36)$ & The Control Group $(n=35 / 40)$ & $t / \chi^{2}$ & $\mathbf{P}$ \\
\hline $\begin{array}{l}\text { Decrease rate of serum } \beta \text {-HCG } 24 \text { hours after } \\
\text { surgery }(\%)\end{array}$ & $81 \pm 2.15$ & $81.40 \pm 2.01$ & -0.81 & 0.42 \\
\hline Length of hospital stay (day) & $5.28 \pm 1.21$ & $5.33 \pm 1.04$ & -0.24 & 0.81 \\
\hline Hospitalization cost (Dollar) & $2800.772 \pm 216.374$ & $3959.807 \pm 325.022$ & -18.086 & $<0.001$ \\
\hline Menelipsis time $(d)$ & $35.89 \pm 5.917$ & $47.20 \pm 18.088$ & -3.581 & 0.001 \\
\hline The incidence of postoperative complications (\%) & $6 \pm 2.320$ & $23 \pm 4.230$ & -2.131 & 0.036 \\
\hline
\end{tabular}

Ovarian Function Indicator Comparisons Between the Two Groups

Preoperative Ovarian Function Indicator

Comparison Between the Two Groups

The preoperative antral follicle count (AFC) and FSH, LH, and

E2 levels were compared between the two groups the differences were not statistically significant $(\mathrm{P}>0.05$, Table 3$)$.

\section{Postoperative Ovarian Function Comparison Between the Two Groups}

Since the patients were in early stages of pregnancy (six to eight weeks) before surgery, the following comparisons are of the postoperative patient ovarian functions (see Appendix A). In general, the postoperative FSH and LH levels of the patients in the control group all increased significantly to different degrees. The overall trend showed that the levels of the two hormones gradually declined to normal levels before six months after surgery; however, there were different degree decreases in the patient E2 levels. With the recovery of ovarian function, the E2 levels gradually returned to normal; however, several patients needed more time for recovery.

Ovarian Function Comparison Between the Two Groups at One Month After Surgery

At 1 month after surgery, the FSH and LH levels were both within the normal range in the study group, while significantly higher than that in control group $(\mathrm{P}<0.001)$. The E2 level was within the normal range in the study group, while lower than normal in the control group; there was a significant difference between the two groups $(\mathrm{P}<0.001)$. There was no significant difference in AFC levels between the two groups $(\mathrm{P}>0.05$, Table 4$)$.

Ovarian Function Comparison Between the Two Groups at Three Months After Surgery

At three months after the operation, the FSH and LH levels were within the normal range in the study group, while still higher than normal in the control group; the differences were statistically significant $(\mathrm{P}<0.001)$. While the $\mathrm{E} 2$ level was within the normal range in the study group, it was lower than normal in the control group; the differences were statistically significant $(\mathrm{P}<0.001)$. There was no significant difference in the AFC level between the two groups $(\mathrm{P}>0.05$, Table 4$)$.

Ovarian Function Comparison Between the Two Groups at Six Months After Surgery

At six months after surgery, the FSH and LH levels were within the normal range in the study group and had returned to the normal level in the control group; there were no significant differences between the two groups $(\mathrm{P}>0.05)$. Furthermore, the E2 level had returned to the normal level in both groups; there was no significant difference between the two groups, as well as the AFC level ( $\mathrm{P}>0.05$, Table 4).

Postoperative Study Group Ovarian Function Comparison The FSH, LH, and E2 levels at one month after surgery were compared with the levels at three months after surgery; the differences were not statistically significant $(\mathrm{P}>0.05)$. As well

Table 3 Comparison of Preoperative Ovarian Function Indicators $(\overline{\mathrm{x}} \pm \mathrm{s})$

\begin{tabular}{|l|l|l|l|l|}
\hline Indicators & The Study Group(n=33/36) & The Control Group $(\mathbf{n = 3 5 / 4 0 )}$ & $\boldsymbol{t}$ & $\boldsymbol{p}$ \\
\hline FSH $(\mathrm{mlU} / \mathrm{mL})$ & $2.719 \pm 1.190$ & $2.470 \pm I .371$ & 0.84 & 0.40 \\
LH $(\mathrm{mlU} / \mathrm{mL})$ & $2.508 \pm 1.304$ & $2.385 \pm 1.452$ & 0.39 & 0.70 \\
E2 $(\mathrm{pmol} / \mathrm{mL})$ & $844.358 \pm 368.031$ & $768.368 \pm 349.942$ & 0.92 & 0.36 \\
AFC & $6.42 \pm 1.481$ & $5.95 \pm 1.339$ & 0.86 & 0.15 \\
\hline
\end{tabular}


Table 4 Comparison of Ovarian Function Between the Two Groups

\begin{tabular}{|c|c|c|c|c|}
\hline Indicators & The Study Group (n=33/36) & The Control Group $(n=35 / 40)$ & $t$ & $p$ \\
\hline \multicolumn{5}{|c|}{ I month after surgery } \\
\hline $\mathrm{FSH}(\mathrm{mlU} / \mathrm{mL})$ & $7.289 \pm 1.035$ & $20.31 \pm 2.742$ & -26.805 & $<0.001$ \\
\hline $\mathrm{LH}(\mathrm{mlU} / \mathrm{mL})$ & $7.397 \pm 1.294$ & $20.240 \pm 3.091$ & -23.151 & $<0.001$ \\
\hline E2 $(\mathrm{pmol} / \mathrm{mL})$ & $143.297 \pm 29.400$ & $103.143 \pm 14.103$ & 7.712 & $<0.001$ \\
\hline AFC & $6.21 \pm 1.29$ & $6.35 \pm 1.29$ & -0.67 & 0.58 \\
\hline \multicolumn{5}{|c|}{3 month after surgery } \\
\hline $\mathrm{FSH}(\mathrm{mlU} / \mathrm{mL})$ & $7.397 \pm 0.868$ & $13.263 \pm 2.089$ & -15.663 & $<0.001$ \\
\hline $\mathrm{LH}(\mathrm{mlU} / \mathrm{mL})$ & $7.800 \pm 1.235$ & $\mid 3.268 \pm 1.88$ & -14.777 & $<0.001$ \\
\hline E2 (pmol/mL) & $145.447 \pm 29.639$ & $119.783 \pm 15.270$ & 4.815 & $<0.001$ \\
\hline AFC & $6.32 \pm 1.24$ & $6.43 \pm 1.43$ & -0.66 & 0.66 \\
\hline \multicolumn{5}{|c|}{6 month after surgery } \\
\hline $\mathrm{FSH}(\mathrm{mlU} / \mathrm{mL})$ & $7.39 \pm 1.31$ & $7.62 \pm 1.06$ & -0.83 & 0.41 \\
\hline $\mathrm{LH}(\mathrm{mlU} / \mathrm{mL})$ & $7.52 \pm 1.32$ & $7.52 \pm 1.28$ & -0.00 & 1.00 \\
\hline E2 (pmol/mL) & $138.28 \pm 26.60$ & $145.43 \pm 25.23$ & -1.20 & 0.23 \\
\hline AFC & $6.08 \pm 1.22$ & $6.30 \pm 1.36$ & -1.63 & 0.24 \\
\hline
\end{tabular}

Abbreviations: FSH, Follicle-stimulating hormone; LH, luteinizing hormone; E2, estradiol; AFC, antral follicle count.

as these indicators no significant differences:between one month and six months after surgery $(\mathrm{P}>0.05)$, between three months and six months after surgery $(\mathrm{P}>0.05)$. The postoperative FSH and LH levels were significantly increased compared with preoperative levels; however, there was no significant change after the normal level was restored, and the pairwise comparison showed no statistically significant differences $(\mathrm{P}>$ 0.05). The postoperative E2 level decreased significantly compared with the preoperative level; however, there was no significant change after the normal range was restored after the operation, and the pairwise comparison showed no statistically significant differences $(\mathrm{P}>0.05)$. There was no significant change in AFC during the postoperative observation, and the differences within the group were not statistically significant $(\mathrm{P}$ $>0.05$, Table 5).

\section{Postoperative Control Group Ovarian Function Comparison}

The FSH, LH, and E2 levels in the control group at one month after surgery were compared with the levels at three months after surgery $(\mathrm{P}<0.001)$; the differences were statistically significant $(\mathrm{P}<0.05)$. There were no statistically significant differences in the $\mathrm{FSH}, \mathrm{LH}$, and E2 levels $(\mathrm{P}<0.05)$ : between one month and six months after surgery $(\mathrm{P}<0.001)$, between three months and six months after surgery $(\mathrm{P}<0.001)$. The FSH and LH levels in the control group increased to a certain extent at one month and three months after surgery, showed a downward trend compared with the last time point, and returned to a normal level at six months after surgery; the differences within the group were statistically significant $(\mathrm{P}<0.05)$.

The control group E2 level decreased at one month and three months after surgery and was essentially lower than the normal level; however, it showed an upward trend compared with the last node and returned to normal at six months after operation; there were significant differences between the groups when compared $(\mathrm{P}<0.05)$, indicating that the patient ovarian function had gradually recovered.

Table 5 Comparison of Ovarian Function in the Study Group After Surgery

\begin{tabular}{|l|c|c|c|c|c|c|}
\hline \multirow{2}{*}{ The Study Group } & \multicolumn{5}{|c|}{ Follow-Up Time (After Surgery) } \\
\cline { 2 - 7 } & \multicolumn{2}{|c|}{ I Month and 3 Months } & I Month and 6 Months & \multicolumn{2}{c|}{ 3 Month and 6 Months } \\
\cline { 2 - 7 } & $\boldsymbol{t}$ & $\boldsymbol{P}$ & $\boldsymbol{t}$ & $\boldsymbol{P}$ & $\boldsymbol{t}$ & $\boldsymbol{P}$ \\
\hline FSH $(\mathrm{mlU} / \mathrm{mL})$ & -0.52 & 0.60 & -0.33 & 0.74 & 0.03 & 0.98 \\
LH (mlU/mL) & -1.29 & 0.20 & -0.42 & 0.68 & 0.85 & 0.40 \\
E2 (pmol/mL) & -0.32 & 0.75 & 0.77 & 0.45 & 1.03 & 0.31 \\
AFC & -0.65 & 0.53 & 0.60 & 0.43 & 0.90 & 0.90 \\
\hline
\end{tabular}

Abbreviations: FSH, Follicle-stimulating hormone; LH, luteinizing hormone; E2, estradiol; AFC, antral follicle count. 
Table 6 Comparison of Ovarian Function in the Control Group After Surgery

\begin{tabular}{|c|c|c|c|c|c|c|}
\hline \multirow[t]{3}{*}{ The Control Group } & \multicolumn{6}{|c|}{ Follow-Up Time (After Surgery) } \\
\hline & \multicolumn{2}{|c|}{ I Month and 3 Months } & \multicolumn{2}{|c|}{ I Month and 6 Months } & \multicolumn{2}{|c|}{3 Month and 6 Months } \\
\hline & $t$ & $\mathbf{P}$ & $t$ & $\mathbf{P}$ & $t$ & $\boldsymbol{P}$ \\
\hline $\mathrm{FSH}(\mathrm{mlU} / \mathrm{mL})$ & 12.46 & $<0.001$ & 26.120 & $<0.001$ & 14.56 & $<0.001$ \\
\hline $\mathrm{LH}(\mathrm{mlU} / \mathrm{mL})$ & 10.93 & $<0.001$ & 25.055 & $<0.001$ & 16.42 & $<0.001$ \\
\hline E2 (pmol/mL) & -4.79 & $<0.001$ & -9.200 & $<0.001$ & -6.276 & $<0.001$ \\
\hline AFC & -0.55 & 0.79 & 0.25 & 0.87 & 0.95 & 0.33 \\
\hline
\end{tabular}

Abbreviations: FSH, Follicle-stimulating hormone; LH, luteinizing hormone; E2, estradiol; AFC, antral follicle count.

The postoperative E2 level change trend was not consistent in the two groups. The E2 change trend in the control group was obvious, and the difference between the two groups was statistically significant $(\mathrm{P}<0.05)$. There was no significant change in the AFC during the postoperative observation; the difference was not statistically significant $(P>0.05$, Table 6$)$.

\section{Discussion}

Due to the implementation of China's two-child policy, the number of patients who underwent a cesarean section with uterus scarring is also increasing. Furthermore, the CSP incidence is rising unavoidably year-by-year. The clinical CSP manifestations lack specificity. Most patients show painless vaginal bleeding after amennorhea, some patients may experience mild abdominal pain or discomfort without vaginal bleeding, and some patients experience both vaginal bleeding and lower abdominal discomfort after menelipsis.

Nowadays, UAE is used for preprocessing in order to prevent intraoperative bleeding in clinical practice; however, considering the cost, impossibility of simultaneous operations, postoperative complications, and psychological effects, many obstetricians and gynecologists are constantly searching for new surgical options. In the present study, laparoscopic TIIAB was used as a preconditioning method. This not only reduced the cost of surgery but could also be completed in one operation without postoperative braking. For this reason, the patient acceptance of this method is high. In our study, the surgery cost, menelipsis time, postoperative complications in the study group are all superior than those in control group. In addition, several studies have reported that intraoperative ligation of bilateral internal iliac arteries followed by local lesion resection can significantly reduce the risk of intraoperative and postoperative bleeding. ${ }^{14}$ These protocols are effective in controlling bleeding from tissue removal and uterine repair, not only because of the advantages of laparoscopic surgery itself, but also the pretreatment can reduce blood supply, as well as the utilization of hemostatic drugs, such as oxytocin and pituitrin. Chen et al adopted TIIAB combined with scar pregnancy lesion resection and uterine repair in the treatment of 12 patients with CSP. This method also achieved good surgical effects, supporting the viewpoints of the present paper. TIIAB and UAE, the two pretreatment can both reduce the intraoperative blood loss by blocking the uterine artery temporarily, the former involves less procedures and reduce the total therapeutic time, which produces less expense. In their study, Cao et a ${ }^{13}$ conducted follow-ups regarding the menstrual recovery of 119 patients with CSP after undergoing UAE + uterine curettage. Among these patients, $58(57.4 \%)$ had hypomenorrhea, $2(1.7 \%)$ had amenorrhea. In this study, menstruation did not resume at three months after surgery in 1 of the 40 patients who underwent UAE. The incidence was $2.5 \%$. The hysteroscopy results showed that the uterine fundus endometrium was thin, the uterine cavity of a cylindric shape, the adhesion of the two side walls close, and the ovarian function decreased to below-normal levels. A total of 2 patients (5\%) developed postoperative fever, and 3 (7.5\%) developed pelvic pain. In their study, Sun et al ${ }^{16}$ evaluate the ovarian function of 374 patients after UAE, finding that the ovarian function was not significantly affected. However, certain literature reports that after UAE, patient ovarian function decreased, the abortion rate increased, and fertility was affected. There was even an occurrence of premature ovarian failure. ${ }^{17}$ Hence, UAE is controversial, as it may affect ovarian function. The FSH, LH, and E2 levels are widely used in ovarian function evaluations (The AMH index was not included in the analysis due to lack of data caused by low patient compliance). Other indicators, including inhibin $\mathrm{B}$, anti-Mullerian hormone, $\mathrm{AFC}$, ovarian volume, and the maximum plane average diameter, ${ }^{18}$ are now more widely applicated in assisted reproductive technology and less used in post-UAE ovarian function evaluations. Therefore, in addition to the FSH, LH, and E2 levels, AFC was selected as a study evaluation factor. The 
FSH and LH levels were higher at one month and three months after UAE than before UAE. However, there was no significant difference in the FSH and LH levels before operation and six months after operation. In addition, the menelipsis time in the study group was significantly shorter than that in the control group; the difference was statistically significant. It can be concluded that UAE has a certain shortterm influence on ovarian function; however, the function gradually returns to normal. UAE is considered to result in a state of relative ovarian ischemia and to reduce ovarian function. UAE leads to an insufficient ovary blood supply; the mechanism may include uterine artery ovarian branch blockage or the diffusion of embolization material into the ovarian vessels through the uterine artery, followed by partial vessel plugging. ${ }^{16,19,20}$ There was no significant decrease in AFC during the post-UAE observation period. The postoperative complications in the study group are obviously less than that in the control group, which is more likely associated with the accurate positioning in the TIIAB surgery. In the UAE operation, due to the individual vessels anatomy differences, the imaging equipment differences, the super selection of uterine vessel, and the positive effect of UAE are more uncertain than TAIIB. The possible reasons for this are: (1) the ultrasound-detected sinus follicles were small anechoic follicles with $2-10-\mathrm{mm}$ diameters that were visible to the gross eye. If sonographers mistake all small anechoic follicles for follicles, an error in the sinus follicle count may occur; (2) in the case of reduced ovarian function, some sinus follicles may stagnate until the next menstrual cycle. This may result in an unchanged follicle count. ${ }^{17}$ In the present study, each patient could not be measured and counted by the same experienced doctor, resulting in certain errors. Simultaneously, it should be noted that post-UAE deep-vein thrombosis has a Meta-analysis incidence of approximately $0.2 \%{ }^{21}$ This is similar to the incidence reported by Toor SS et al.. ${ }^{22}$ Besides, the use of UAE therapy $(\mathrm{OR}, 5.796 ; \mathrm{p}=0.003)$ is a CSP recurrence risk factor. ${ }^{23}$

\section{Shortcomings and Prospects}

In recent years, CSP has been increasingly attracting the attention of obstetricians and gynecologists due to its bleeding risk. We should be vigilant about CSP in clinical practice. If surgical treatment is required, the pregnancy at the scar should be completely removed during the surgery in order to avoid residue. At present, the vast majority of patients with CSP can retain the uterus after treatment; however, there is a lack of statistical studies on the impact of CSP on the reproductive function. The preservation of the reproductive function, the surgical skills of surgeons, and the clinical manifestations of patients are still important factors that are taken into consideration when forming a surgical plan. Ben Nagi J et $\mathrm{al}^{24}$ shows that reproductive outcomes following treatment of caesarean scar ectopic pregnancies are favourable. The risk of complications including recurrent scar implantation appears to be low. The lack of studies including greater sample sizes and longer follow-up periods makes it difficult to develop guidelines for evidencebased medicine. As this study did not follow up on the patients' pregnancy situations after surgery, it is impossible to determine the treatment's influence on the pregnancy rate. There is still little evidence that patients benefit from TIIAB. Protecting women's ovarian functions is of great importance. However, since the ovaries are supplied by both the ovarian artery and ovarian uterine artery branch, it is uncertain whether TIIAB affects the ovarian blood supply and causes premature ovarian failure. Studies have shown that ovarian function is not affected after uterine artery occlusion. ${ }^{25-28} \mathrm{We}$ consider TIIAB to be able to restore ovarian blood vessels without affecting the ovarian function. However, the evidence determining that TIIAB can help avoid complications, such as impairing patient ovarian function, is still insufficient. Moreover, long-term evidence-based medical evidence is required in order to prove that TIIAB is extremely safe and effective. In addition, the technique of temporarily blocking the iliac artery can be applied during the operation; it is a good method of preventing serious intraoperative bleeding, such as angular pregnancy, interstitial tubal pregnancy, hysteromyoma, and uterine adenomyoma. Especially in trophoblastic tumor resections, operation is more suitable, as the lesions can easily bleed, and oxytocin or pituitrin cannot be applied during the operation. Laparoscopic TIIAB can reduce postoperative complications and hospitalization costs, thus benefiting most patients. However, there is still a long way to go for the clinical application of TIIAB, and new evidence is needed in order to promote its future application. The small sample size may weaken the generalisability of the results, and the conclusions still need to be supported by data after larger sample studies.

\section{Conclusions}

Compared with UAE, laparoscopic TIIAB has the advantages of a low hospitalization cost, lower postoperative complication rate, and shorter menelipsis time for the patients with type III 
CSP. Moreover, it avoids ovarian function damage. TIIAB is a safe method worthy of clinical popularization.

\section{Ethics Approval and Consent to Participate}

This study was conducted in accordance with the declaration of Helsinki. This study was conducted with approval from the Ethics Committee of The First Affiliated Hospital of Zhengzhou University. A written informed consent was obtained from all participants.

\section{Disclosure}

The authors report no conflicts of interest in this work.

\section{References}

1. Xie X, Kong B, Duan T. Obstetrics and Gynecology. People's Medical Publishing House. Beijing, China; 2018.

2. Birch Petersen K, Hoffmann E, Rifbjerg Larsen C, Svarre Nielsen H. Cesarean scar pregnancy: a systematic review of treatment studies. Fertil Steril. 2016;105(4):958-967. PMID: 26794422. doi:10.1016/j. fertnstert.2015.12.130

3. Larsen JV, Solomon MH. Pregnancy in a uterine scar sacculusan unusual cause of postabortal haemorrhage. A case report. $S$ Afr Med J. 1978;53(4):142-143.

4. Ash A, Smith A, Maxwell D. Caesarean scar pregnancy. BJOG. 2007;114:253-263. doi:10.1111/j.1471-0528.2006.01237.x

5. Parker VL, Srinivas M. Non-tubal ectopic pregnancy. Arch Gynecol Obstet. 2016;294:19-27. doi:10.1007/s00404-016-4069-y

6. Li Y, Gong L, Wu X, Gao H, Zheng H, Lan W. Randomized controlled trial of hysteroscopy or ultrasonography versus no guidance during D\&C after uterine artery chemoembolization for cesarean scar pregnancy. Int J Gynaecol Obstet. 2016;135(2):158-162. doi:10.1016/j.ijgo.2016.04.019

7. Koplay M, Dogan NU, Sivri M, Erdogan H, Dogan S, Celik C. Ectopic pregnancy in a cesarean section scar: successful management using vacuum aspiration under laparoscopic supervision-mini review of current literature. Case Rep Surg. 2016;2016:7460687. doi:10.1155/2016/7460687

8. Le A, Shan L, Xiao T, Zhuo R, Xiong H, Wang Z. Transvaginal surgical treatment of cesarean scar ectopic pregnancy. Arch Gynecol Obstet. 2013;287(4):791-796. doi:10.1007/s00404-012-2617-7

9. Glenn TL, Bembry J, Findley AD, et al. Cesarean scar ectopic pregnancy: current management strategies. Obstet Gynecol Surv. 2018;73(5):293-302. doi:10.1097/OGX.0000000000000561

10. Fylstra DL, Pound-Chang T, Miller MG, Cooper A, Miller KM. Ectopic pregnancy within a cesarean scar: a review. Am J Obstet Gynecol. 2002;187(2):302-304. doi:10.1067/mob.2002.125998

11. Miller R, Timor-Tritsch IE, Gyamfi-Bannerman C. Society for Maternal-Fetal Medicine (SMFM) consult series \#49: cesarean scar pregnancy. Am J Obstet Gynecol. 2020;222(5):B2-B14. doi:10.1016/ j.ajog.2020.01.030

12. Liang F, He J. Methotrexate-based bilateral uterine arterial chemoem- bolization for treatment of cesarean scar pregnancy. Acta Obstet Gynecol Scand. 2010;89(12):1592-1594. doi:10.3109/00016349.2010.512973
13. Cao GS, Liu RQ, Liu YY, et al. Menstruation recovery in scar pregnancy patients undergoing UAE and curettage and its influencing factors. Medicine. 2018;97(11):e9584. doi:10.1097/ MD.0000000000009584

14. Li Y, Zhou Q, Wang SX. Diagnosis and treatment of 29 patients with scar pregnancy during cesarean section. Chin $J$ Obstet Gynecol. 2012;13(06):409-412.

15. Dubois J, Garel L, Grignon A, Lemay M, Leduc L. Placenta percreta: balloon occlusion and embolization of the internal iliac arteries to reduce intraoperative blood losses. Am J Obstet Gynecol. 1997;176 (3):723-726. doi:10.1016/S0002-9378(97)70582-9

16. Sun ZT, Zhang L, Liu W, Li JJ, Wang YC. Clinical value of uterine artery embolization before curetection in the treatment of 347 patients with uterine scar pregnancy. Chin J Interv Radiol. 2018;6 (03):204-208.

17. Zhou CJ, Yang N, Liu XW, Zhao H, Gong XL. Evaluation of the effect of uterine artery embolization on ovarian function by transvaginal color doppler ultrasound. Med Health Equip. 2019;40 (07):58-61.

18. Gong XJ. Effect of Uterine Artery Embolization on Ovarian Function. Tianjin: Tianjin Medical University; 2019.

19. Czuczwar P, Stepniak A, Milart P, Paszkowski T, Wozniak S. Comparison of the influence of three fibroid treatment options: supracervical hysterectomy, ulipristal acetate and uterine artery embolization on ovarian reserve - an observational study. $J$ Ovarian Res. 2018;11(1):45. doi:10.1186/s13048-018-0420-1

20. Jung DC, Kim MD, Oh YT, Won JY, Lee DY. Prediction of early response to uterine arterial embolisation of adenomyosis: value of T2 signal intensity ratio of adenomyosis. Eur Radiol. 2012;22 (9):2044-2049. doi:10.1007/s00330-012-2436-z

21. Lee MS, Kim MD, Lee M, et al. Contrast-enhanced MR angiography of uterine arteries for the prediction of ovarian artery embolization in 349 patients. $J$ Vasc Interv Radiol. 2012;23(9):1174-1179. doi:10.1016/j.jvir.2012.06.015

22. Toor SS. Complication rates and effectiveness of uterine artery embolization in the treatment of symptomatic leiomyomas: a systematic review and meta-analysis. Am J Roentgenol. 2012;199 (5):1153-1163. doi:10.2214/AJR.11.8362

23. Nusrathi A, Lin KQ, Lin J. Analysis of the influencing factors of uterine artery embolization combined with uterine debridement for treatment of massive vaginal bleeding in cesarean scar pregnancy. Chin J Fam Plann. 2016;24(12):828-831.

24. Ben Nagi J, Helmy S, Ofili-Yebovi D, Yazbek J, Sawyer E, Jurkovic D. Reproductive outcomes of women with a previous history of caesarean scar ectopic pregnancies. Hum Reprod. 2007;22 (7):2012-2015. doi:10.1093/humrep/dem078

25. Timor-Tritsch IE, Monteagudo A, Calì G, D’Antonio F, Kaelin Agten A. Cesarean scar pregnancy: diagnosis and pathogenesis. Obstet Gynecol Clin North Am. 2019;46(4):797-811. doi:10.1016/j. ogc.2019.07.009

26. Yang SP. Effect of laparoscopic uterine artery occlusion on ovarian function and fertility in patients with uterine fibroids. Chin J Clin. 2019;47(03):346-348.

27. Chen JL, Liu J, Mu PH, Deng L, Zhang H. Analysis of the application value of temporary uterine artery occlusion in laparoscopic myomectomy. Hebei Med. 2014;20(09):1452-1455.

28. Yin CH, OuYang SL, Y1 L, Ss L, He LS. Clinical effect of laparoscopic temporary occlusion of uterine artery in patients undergoing myomectomy. Med Equip. 2017;30(05):87-88. 


\section{Publish your work in this journal}

The International Journal of General Medicine is an international, peer-reviewed open-access journal that focuses on general and internal medicine, pathogenesis, epidemiology, diagnosis, monitoring and treatment protocols. The journal is characterized by the rapid reporting of reviews, original research and clinical studies

across all disease areas. The manuscript management system is completely online and includes a very quick and fair peer-review system, which is all easy to use. Visit http://www.dovepress.com/ testimonials.php to read real quotes from published authors. 